\title{
Estimulación medular modulada automáticamente. Evidencia de los sistemas de estimulación cerrada: hacia el marcapasos del dolor
} Spinal stimulation modulated automatically. Evidence of closed stimulation systems: towards the pain pacemaker

\section{Abejón González}

Director del Departamento de Unidad del Dolor. Hospital Universitario Quirón. Madrid. Profesor asociado de la Universidad Europea de Madrid, España

La estimulación de cordones posteriores (SCS) se lleva empleando en el tratamiento del dolor desde hace más de 50 años con grandes avances en la terapia, aunque con dificultad para poder conocer el verdadero mecanismo de acción, y con el desarrollo de diferentes sistemas, aunque todos con la misma filosofía. El avance en los dispositivos de neuroestimulación y las diferentes modalidades de estimulación han mejorado y ampliado su utilidad bajos las adecuadas normas de aplicación recomendadas [1].

La SCS tiene reconocidas indicaciones en el tratamiento del dolor por polineuroptía diabética, cirugía fallida de espalda y dolor regional complejo. También se ha empleado en otras indicaciones de dolor con menor evidencia (1). En España, las unidades del dolor atienden un 18,2 \% de casos de lumbociática crónica y un 4,3 de casos de cirugía de espalda fallida [2].

Los resultados globales de la SCS en cirugía fallida de espalda, según la evidencia de más calidad, beneficia al $50 \%$ de los pacientes, con el criterio de un alivio del dolor del $50 \%$ (3); por tanto, de carácter relevante. No obstante, las fluctuaciones en la percepción de parestesias son causadas en gran medida por la variación en la distancia entre los electrodos fijos y la médula espinal, como consecuencia del movimientos voluntarios o involuntarios del paciente con sub o hiperestimulación. Los pacientes emplean múltiples estrategias con diferentes éxitos para manejar las fluctuaciones relacionadas con la posición en la percepción de estimulación, lo que puede resultar en la administración de una terapia subóptima [4].

Mekhail y el grupo del estudio Evoke publican en Lancet neurology un valioso estudio a 12 meses, aleatorizado, doble ciego, en 67 pacientes por brazo con dolor lumbar y de extremidades inferiores, comparando la SCS con un sistema de estimulación abierto y salida fija frente a un sistema de estimulación cerrada y salida automáticamente modulada [5].
La estimulación de la médula espinal de circuito abierto y salida fija ofrece una salida fija de corriente de estimulación. Los sistemas de estimulación de la médula espinal disponibles actualmente no miden una respuesta neurofisiológica a la estimulación y, por lo tanto, se desconoce la activación de la médula espinal. En la estimulación de la médula espinal de circuito cerrado, la corriente de estimulación se ajusta automáticamente en tiempo real a través de la retroalimentación de la respuesta neurofisiológica medida (mediante el registro de ECAP = potencial de acción compuesto evocado] a la estimulación para mantener la respuesta objetivo. Por lo tanto, el objetivo de la estimulación de la médula espinal de asa cerrada es igualar los requisitos del paciente para producir una activación constante.

Los resultados de este estudio ponen de manifiesto una disminución superior en el dolor global de la estimulación de circuito cerrado (ECC) del 72,3\% $[29,0)$ vs. la estimulación de circuito abierto (ECA), 56,2 \% $[38,5]$ a 12 meses, y similar a los 3 meses, con un grado de disminución del dolor similar a nivel lumbar y en EEll. En cuanto al porcentaje de pacientes con un alivio del $50 \%$, este tuvo lugar el $83 \%$ en el grupo ECC y el $61 \%$ en el grupo SCA. El resto de variables evaluada, de función, calidad de vida y estado emocional, fueron mejores con el sistema ESC.

Los autores vuelven a presentar un estudio de no inferioridad en 3 y 12 meses, con pacientes que representan la causa más frecuente de implantes, los pacientes con dolor en la zona lumbar y en piernas, que han sido refractarios a diferentes tratamientos. Los resultados del estudio presentan una mejoría más importante en los pacientes que emplearon el sistema de circuito cerrado frente a los pacientes en los que se implantó el sistema de circuito abierto o el sistema "convencional", como se podía prever, sin ninguna diferencia en las complicaciones, siendo la 
más frecuente, como en otros estudios, la migración de los electrodos.

Como digo, el sistema de circuito cerrado era previsiblemente mejor por el control a tiempo real de la intensidad de la estimulación, según datos previos [6]. El sistema no solo controla los cambios posturales, sino que realiza la activación del sistema dependiendo de los potenciales evocados, controlando de esta manera todos los cambios que se producen con respecto a una señal electromiografía. Del sistema que nos presentan puede representar una nueva forma de estimulación con un registro a tiempo real de los cambios que se producen, con el consiguiente ahorro de energía y el confort del enfermo implantado. El estudio observacional AVALON, con este sistema de ESC, ha desmontado su utilidad [7], pero el estudio EVOKE confirma con la evidencia de mejor calidad la eficacia real y superior a la estimulación convencional.

\section{BIBLIOGRAFÍA}

1. Deer TR, Krames E, Mekhail N, Pope J, Leong M, StantonHicks $M$, et al. Neuromodulation Appropriateness Consensus Committee. The appropriate use of neurostimulation: new and evolving neurostimulation therapies and applicable treatment for chronic pain and selected disease states. Neuromodulation Appropriateness Consensus Committee.
Neuromodulation. 2014;17(6):599-615. DOI: 10.1111/ ner.12204. PubMed PMID: 25112892.

2. Montero Matamala A, Samper Bernal D, Vidal Fuentes J, Rodríguez Dinten MJ, Jiménez Cosmes L. Estudio para evaluar el perfil del paciente que acude en primera visita a Unidades de Dolor de centros hospitalarios españoles (estudio PANDHORA). Rev Soc Esp Dolor. 2011;18(4):219-27.

3. Taylor RS, Desai MJ, Rigoard P, Taylor RJ. Predictors of pain relief following spinal cord stimulation in chronic back and leg pain and failed back surgery syndrome: a systematic review and meta-regression analysis. Pain Pract. 2014;14(6):489505. DOl: 10.1111/papr.12095.

4. Ross E, Abejón D. Improving patient experience with spinal cord stimulation: implications of position-relatied changes in neurostimulation. Neurmodulation. 2014;17(Supl. 1):36-41. DOI: 10.1111/j.1525-1403.2011.00407.x.

5. Mekhail N, Levy RM, Deer TR, Kapural L, Li S, Amirdelfan $\mathrm{K}$, et al. Evoke Study Group. Long-term safety and efficacy of closed-loop spinal cord stimulation to treat chronic back and leg pain (Evoke): a double-blind, randomised, controlled trial. Lancet Neurol. 2020;19(2):123-34. DOI: 10.1016/ S1474-4422(19)30414-4.

6. Schultz DM, Webster L, Kosek P, Dar U, Tan Y, Sun M. Sensor-driver position adapive spinal cord stimulation for chronic pain. Pain Physician. 2012;15(1):1-12.

7. Russo M, Cousins MJ, Brooker C. Effective relief of pain and associated symptoms with close-loop spinal cord stimulation system: preliminary results of the Avalon study Neuromodulation. 2018;21(1):38-47. 10.1111/ner.12684. 